\title{
Agile MPC system linking manufacturing and market strategies
}

\author{
Ahmed M. Deif*, Waguih H. ElMaraghy \\ Department of Industrial Manufacturing Systems, University of Regina, Regina, Canada
}

\begin{abstract}
A B S T R A C T
Increasing complexity and interdependency in manufacturing enterprises require an agile manufacturing paradigm. This paper considers a dynamic control approach for linking manufacturing strategy with market strategy through a reconfigurable manufacturing planning and control (MPC) system to support agility in this context. A comprehensive MPC model capable of adopting different MPC strategies through distributed controllers of inventory, capacity, and WIP is presented. A hierarchical supervisory controller (referred to as decision logic unit, DLU) that intakes the high-level strategic market decisions and constraints together with feedback of the current manufacturing system state (WIP, production, and inventory levels) and optimally manages the distributed controllers is introduced. The DLU architecture with its three layers and their different functionalities is discussed showing how they link the higher management level to the operational level to satisfy the required demand. A case study for an automatic PCB assembly factory is implemented to demonstrate the applicability of the whole approach. In addition, a comparative cost analysis study is carried out to compare between the developed agile MPC system and classical-inventory- and capacity-based MPC policies in response to different demand patterns. Results showed that the developed agile MPC policy is as cost effective as the inventory-based MPC policy in demand patterns with steady trends, as cost effective as capacity-based MPC in turbulent demand patterns, and far superior than both classical MPC polices in mixed-demand patterns.
\end{abstract}

\section{Introduction}

The need for alignment between market strategy and manufacturing strategy is well established in the literature. Kotha and Swamidass [16] found that a fit between certain dimensions of market strategy and advanced manufacturing technology was associated with superior performance. Hayes and Wheelwright [11] argued that the alignment between manufacturing strategy and market strategy is one dimension of consistency for manufacturing strategy. Hayes and Schmenner [10] contended that "manufacturing functions best when its facilities, technology, and policies are consistent with recognized priorities of corporate strategy". Anderson et al. [1] have stated that proper strategic positioning or aligning of operations capabilities can significantly impact the competitive strength and business performance of an enterprise. Likewise, Skinner [23], using several case studies, has demonstrated that in enterprises where functional (like manufacturing) strategies are in consonance with market strategy, performance is superior to organizations where functional strategies and the market strategy are not aligned. Agile enterprises should thus demonstrate a clear link between both strategies.

\footnotetext{
* Corresponding author. Tel.: +1 5192533000

E-mail address: deif@uwindsor.ca (A.M. Deif).
}

The consistency of the market and manufacturing strategies is important to the role of the manufacturing planning and control (MPC) system within an agile enterprise, as the MPC system is recognized as one of the pivotal infrastructures that firmly support the organization's manufacturing to align with its higher-level market strategy [26]. Today's MPC strategies can be generally classified into two main categories. The first category is the inventory-based MPC system where the inventory and the workin-process (WIP) levels are the main parameters considered for planning and control. The second category is the capacity-based MPC system where the capacity and WIP levels are the main considered parameters. Each MPC strategy has its own merits to buffer against demand uncertainties, with particular demand patterns depending on the business strategy of the enterprise. However, there does not exist an MPC model that manages to adopt both policies to guarantee an effective response to any demand pattern and full alliance with the market strategy, which are major requirements for an enterprise to achieve agility.

This paper presents a dynamic MPC model using distributed controllers that have the ability to control the three parameters (inventory, capacity, and WIP) and thus adopt different manufacturing strategies based on the market strategy of the enterprise. This is achieved through a supervisory controller that controls these distributed controllers for each of the three parameters. The supervisory controller (referred to as the decision logic unit) intakes the high-level market strategy and constraints together with a 
feedback of the current manufacturing system state and optimally adapts the manufacturing system to the optimal suitable operation policy at these conditions.

\section{Literature review}

The application of control-theoretic approaches in manufacturing systems have been excessively researched on the operational and machine levels but not as much on the system and enterprise levels. These approaches are important in the agile manufacturing paradigm because agile manufacturing systems need to be dynamic and controllable to achieve agility objectives. Distributed control is one of the control theory approaches that have the potential to model, analyze, and control agile manufacturing through a network of interconnected controllers. Previous work showed the application of a distributed control approach to traditional manufacturing systems on both an operational level as well as a system level.

Examples of distributed control applications on the operational level include Duffie, Prabhu, and Kaltjob [7], where they solved the problem of controlling arrival times in heterarchical manufacturing systems by modeling and developing a system where the dynamics of autonomous controllers and the physical interactions between entities in the system combine to create system behavior that is seemingly chaotic, but favorable. In similar heterarchical manufacturing systems, Prabhu [21] developed a model of distributed cooperative control that was generic and characterized dynamics of job shops with multiple processing steps and parallel dissimilar machines. Global stability conditions for such nonlinear control systems have been established using results from the Lyapunov theory. Wysk and Smith [31] developed a formal functional characterization of the general shop floor control problem in discrete manufacturing that incorporates different distributed controllers for workstations and their equipment on the shop floor. The control approach attempts to control the planning, scheduling, and execution activities on the shop floor based on process planning. The previous approach was modified and applied with the aid of simulation to control hybrid manufacturing systems in MorenoLizaeanzu et al. [17]. A distributed control approach has been also used for a manufacturing systems scheduling problem Ioannidis et al. [12]. A set of fuzzy controllers has been derived to reduce WIP and synchronize the production system's operation. Tsourveloudis, and Valavanis et al. [25] extended the previous work and developed a two-level control architecture with a supervisory controller at the higher level of production used to tune the operation of the lower-level distributed fuzzy controllers. Bruccoleri, Amico and Perrone [2] proposed a distributed intelligent control approach, which is an agent-based system, to deal with "out of the ordinary" events in the production process in reconfigurable manufacturing systems. The approach relied on the reconfiguration ability of the system to maintain the production flow in such events. The previous approach was extended to include other production scenarios in [3].

Various researches attempted to manipulate a distributed control approach on a system level to control inventory, WIP, and capacity (the main manufacturing control parameters) in manufacturing systems through modeling controllers for each of these parameters. Examples include the APIOBPCS (Automatic Pipeline, Inventory and Order-Based Production Control System) model developed by John, Towill, and Naim [13] used to control supply chain management as in [6], inventory control as in [24], and aggregate planning as in [5]. Also, the funnel model of manufacturing systems developed by Wiendahl [27] is another model that adopted the same control approach with the help of logistic operating curves developed by Nyhuis [20] to control WIP and backlog in manufacturing systems in $[28,29]$. Another single- workstation model developed by Duffie and Falu [8] for closedloop PPC was used to control WIP and backlog in discrete and continuous time domains in [22] and [14], respectively, and was extended to multiple workstations in [15].

The reviewed literatures show that the previous distributed control approaches focused only on events occurring on the shop floor (from an operational or system perspective) and how to control them; thus, in an agile enterprise context there is a need to link this manufacturing level with the high marketing level in a seamless way. Such a link will maintain the alignment between the strategies in both levels, as discussed earlier. In addition, it is shown from an MPC standpoint that these approaches were able only to focus on either one strategy (like capacity-based, WIP-based, or inventory-based strategy) or managed to combine two of them together, but none managed to deal with all three strategies simultaneously. To deal with different market strategies and various demand patterns, there is a need to have an MPC model that is able to control the three parameters and in turn adopt different MPC strategies based on the market need. In this paper, a dynamic MPC model is presented to address these two needs in an agile enterprise.

\section{Agile MPC model}

The dynamic modeling of the agile MPC system aims at constructing a model in which different planning and control configurations can be realized with respect to a higher-level market strategy. The system model shown in Fig. 1 includes the three main controlled variables that can work individually or two at a time based on the decision of the decision logic unit to determine the desired production rate (DPR). The variables are work in process (WIP), capacity rate of the system, and finished inventory level.

The proposed general structure of the agile MPC system can be described as being composed of two main operational layers in addition to a decision logic unit that links these two layers with the higher management layer. The first operation layer is the default (or servo control) layer where the control of the manufacturing system is only based on controlling the WIP level by observing the current level with the target level (based on Little's law). The other layer (intelligent control layer) involves two controllers, the first being an inventory controller that compares the inventory level with the required level based on the service level offered to the customers, and the second being a capacity controller that ensures that the production rate matches the demand (or the order rate). Either can work with the servo control layer or by itself, creating five different MPC configurations or strategies: WIP-based policy, capacity-based policy, inventory-based policy, capacity/WIP-based policy, and inventory/WIP-based policy.

The selection between these strategies is decided by the DLU through engaging and disengaging the controllers of each policy. Furthermore, the DLU provides the system with the reference control points and the updates of the order rate $(\mathrm{OR})$ and shipment time $\left(T_{\mathrm{ST}}\right)$ based on demand data from the higher management level, and at the same time collects all of the data of the current system to help in deciding the optimal MPC configuration.

The production process is modeled as a pipeline where the outflow is lagged by the production lead time, $T_{\mathrm{LT}}$. Simulation results of similar production systems showed an exponential pipeline lag used in this model to be an appropriate compromise between complexity and accuracy [30]. In addition, an exponential delay is also assumed for capacity installation/uninstallation time, $T_{D}$, because capacity scalability cannot be assumed to happen instantaneously.

The main purpose of any manufacturing planning and control system is to set plans and to group control actions to adjust 


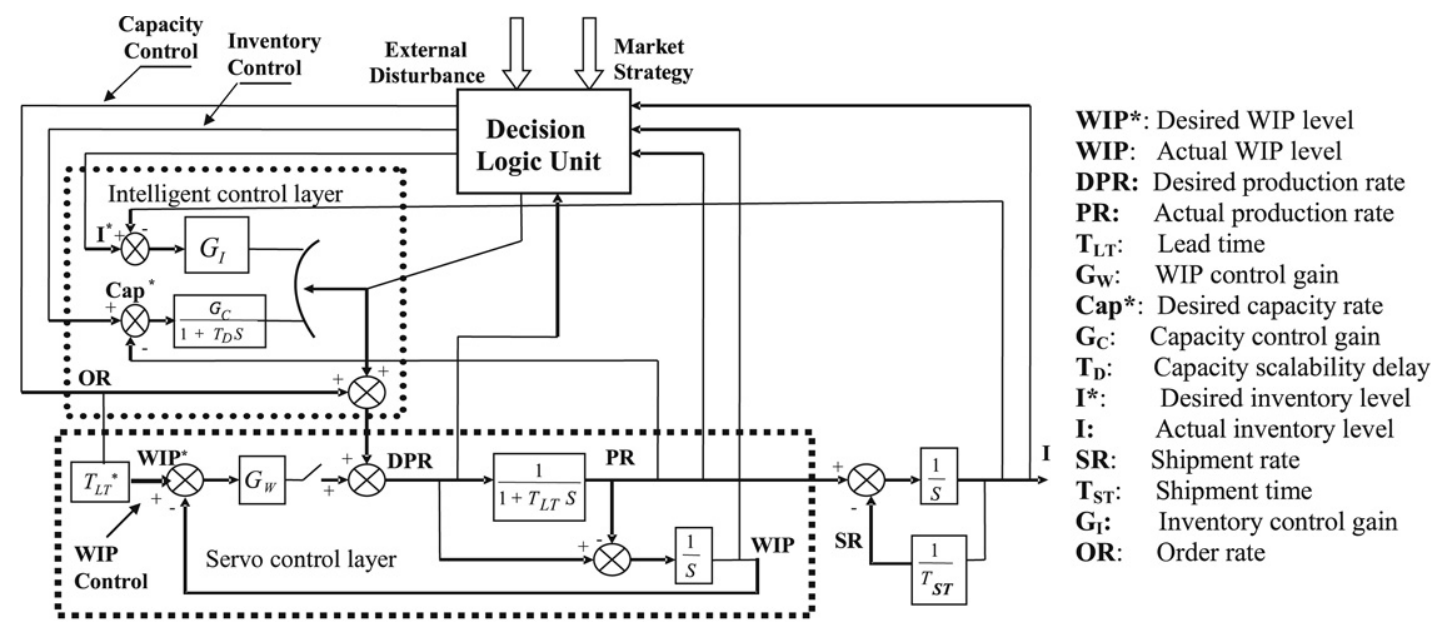

Fig. 1. Agile MPC system model.

the desired production rate (DPR) to meet the demand patterns specified by marketing. In the proposed model, DPR is the sum of the expected losses (which in this case are the expected order rates, OR) plus adjustments in the production-rate level. The adjustments can be in the WIP level, in the actual production-rate (PR) level, in the finished inventory level, or any combination of the previous parameters based on the MPC policy selected by the DLU. The adjustments to the DPR are actually the values of the different controllers' gains in each policy.

It is important also to note that the proposed approach has a continuous time model while its control is a discrete-action one. The continuous time modeling is justified due to the level of abstraction of the model that deals with the tactical rather than the operational level.

Eqs. (1)-(4) list the transfer functions derived for the developed agile MPC system configurations. Without losing the generality, two basic assumptions were made. First, the expected lead time is assumed to be equal to the actual one $\left(T_{\mathrm{LT}}=T_{\mathrm{LT} *}\right)$. Second, the shipment rate is set to be equal to the order rate ( $S R=O R)$. These assumptions are made only for a better understanding of the problem. The proposed model does not have any limitations with respect to considering the case of any linear or nonlinear relation between the variables. More details of the model and its analysis can be found in [4].

\section{Capacity-based MPC system}

$$
\frac{\mathrm{PR}}{\text { Cap }^{*}}=\frac{G_{C} T_{\mathrm{LT}}^{-1} T_{D}^{-1}}{S^{2}+S\left(T_{\mathrm{LT}}^{-1}+T_{D}^{-1}\right)+\left(1+G_{C}\right) T_{\mathrm{LT}}^{-1} T_{D}^{-1}} .
$$

\section{Finished-inventory-based MPC system}

$\frac{I}{I^{*}}=\frac{G_{I} T_{\mathrm{LT}}^{-1}}{S^{2}+S\left(T_{\mathrm{LT}}^{-1}+T_{\mathrm{ST}}^{-1}\right)+G_{I} T_{\mathrm{LT}}^{-1}}$.

\section{Capacity/WIP-based MPC system}

$$
\frac{\mathrm{PR}}{\mathrm{Cap}^{*}}=\frac{G_{W}\left(T_{D}^{-1}+S\right)+G_{C} T_{\mathrm{LT}}^{-1} T_{D}^{-1}}{S^{2}+S\left(T_{D}^{-1}+T_{\mathrm{LT}}^{-1}+G_{W}\right)+\left(G_{W} T_{\mathrm{LT}}+G_{C}+1\right) T_{\mathrm{LT}}^{-1} T_{D}^{-1}} .
$$

\section{Finished inventory/WIP-based MPC system}

$$
\frac{I}{I^{*}}=\frac{G_{I} T_{\mathrm{LT}}^{-1}}{S^{2}+S\left(G_{W}+T_{\mathrm{LT}}^{-1}+T_{\mathrm{ST}}^{-1}\right)+\left(G_{I} T_{\mathrm{LT}}^{-1}\right)} .
$$

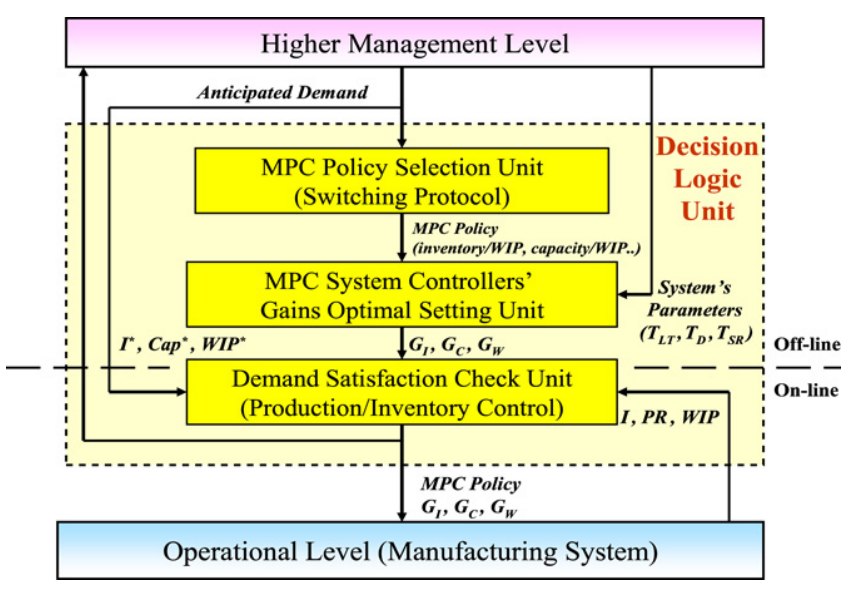

Fig. 2. Architecture of proposed decision logic unit (DLU).

\section{Architecture for the DLU}

Fig. 2 shows the architecture of the DLU of the developed agile MPC system. The figure shows how the DLU unit links the higher management level with the operational level (manufacturing system), which is a basic requirement to realize agility in any manufacturing corporation. Such a detailed link was always missing in previous MPC research work. The architecture of the DLU is composed of three hierarchical layers. The first two layers function off-line, and the third layer is an on-line control layer. The units in each of the three layers are explained in the following sections.

\section{MPC policy selection unit}

This unit is responsible for analyzing the anticipated demand profile by the higher management level and its marketing strategy. Based on the analysis of the demand profile, the unit decides which policy (or MPC strategy) is to be adopted over which interval of time of that expected demand. In other words, the output would be a plan that indicates which MPC policy (inventory/WIP, capacity/WIP, capacity, or inventory) is to be applied during which months of the year (if the demand profile was anticipated monthly). It is important to note that this unit can deal with sudden changes in the anticipated demand. Such an ability is very important in agile manufacturing environments. From a control perspective, this selection process can be considered to be the switching protocol that governs the engagement and 
Table 1

Natural frequency and damping ratio of the different agile MPC systems policies

\begin{tabular}{|c|c|c|}
\hline MPC system policy & Natural frequency & Damping ratio \\
\hline Inventory & $\omega_{n}=\sqrt{\frac{G_{I}}{T_{\mathrm{LT}}}}$ & $\xi=\frac{1}{2 \omega_{n}}\left(\frac{1}{T_{\mathrm{LT}}}+\frac{1}{T_{\mathrm{SR}}}\right)$ \\
\hline Capacity & $\omega_{n}=\sqrt{\frac{1+G_{C}}{T_{\mathrm{LT}} T_{D}}}$ & $\xi=\frac{1}{2 \omega_{n}}\left(\frac{1}{T_{\mathrm{LT}}}+\frac{1}{T_{D}}\right)$ \\
\hline Inventory/WIP & $\omega_{n}=\sqrt{\frac{G_{l}}{T_{\mathrm{LT}}}}$ & $\xi=\frac{1}{2 \omega_{n}}\left(\frac{1}{T_{\mathrm{LT}}}+\frac{1}{T_{\mathrm{SR}}}+G_{W}\right)$ \\
\hline Capacity/WIP & $\omega_{n}=\sqrt{\frac{G_{W} T_{L T}+G_{C}+1}{T_{L T} T_{D}}}$ & $\xi=\frac{1}{2 \omega_{n}}\left(\frac{1}{T_{\mathrm{LT}}}+\frac{1}{T_{D}}+G_{W}\right)$ \\
\hline
\end{tabular}

disengagement of the distributed controllers involved in the developed MPC system.

The switching algorithm (based on segmentation) receives the set of anticipated demand data from the higher management level and starts with the first three points (or months) and tests the absolute error of these points with their calculated regression line. If the error is relatively small, this means that the demand within this range is of a steady trend, and thus an inventory-based policy is selected. On the other hand if this error shows high values, this means that the demand experiences great variations and, thus, a capacity-based policy is better to hedge against these variations in this demand period. It is important to note that the value of the error in this algorithm depends on the degree of market sensitivity the enterprise would like to have in its marketing strategy.

After the decision is taken for the first three demand data, the algorithm will check the next two data with the last point of the previously tested three points, and the same regression analysis is carried out. The analysis will keep on exploring the demand data until the whole planning period (all anticipated demands) is covered and divided into different regions, where a specific MPC policy is applied to each region.

\section{MPC controllers' gains setting unit}

This can be considered the heart of the developed DLU. This unit is responsible for deciding on the optimal values of the distributed controllers' gains in the developed MPC system. By "optimal" it is meant the value of the gains that will satisfy the competing agility objectives of responsiveness and cost effectiveness.

From a dynamic analysis standpoint, the responsiveness of the developed dynamic system can be expressed by the rise time [Eq. (5)]. By definition, this is the time it takes the system to rise from $10 \%$ to $90 \%$ of its target value [19]. This measure can be used as an indicator of how fast the system can respond to $90 \%$ of the required demand and, therefore, the degree of its responsiveness.

$t_{\Gamma, 10,90 \cong}=\frac{0.8+2.5 \xi}{\omega_{n}} \quad 0 \leq \xi \leq 1$.

In addition, one of the cost aspects of production can be reflected in the value of the production overshoot measure [Eq. (6)]. The maximum overshoot, or sometimes called the percent overshoot, is the amount that the waveform overshoots the steadystate, or final, value of the time required to reach maximum peak, expressed as a percentage of the steady-state value [19]. This measure directly describes the maximum amount of excess production the system will encounter to respond to a sudden change in demand.

$\% O S=\mathrm{e}^{-\left(\xi \pi / \sqrt{1-\xi^{2}}\right)} * 100$.

The objective function (7) thus will aim to minimize the rise time and at the same time minimize the production overshoot. Each objective will take a specific weight, a, based on the strategy of the higher-level management as mentioned before. This unit is responsible for that multi-objective optimization activity. As for the constraints, they are mainly the stability constraints and the manufacturing system's constraints on capacity and input rate.

Min $: \alpha\left(\frac{0.8+2.5 \xi}{\omega_{n}}\right)+(1-\alpha)\left(\mathrm{e}^{-\left(\xi \pi / \sqrt{1-\xi^{2}}\right)} * 100\right)$.

To calculate the values of the parameters of Eq. (7), Table 1 shows the natural frequency and damping ratio of different MPC strategies or policies. From the table, one can realize that the optimization process is a function of the manufacturing time-based parameters (lead time, scalability delay time, and shipment time), and thus it can be altered (or changed) based on strategic decisions from the higher marketing level to invest in the manufacturing system or change market policy in order to change these parameters and, in turn, change the values of the controllers' gains.

The MPC system controllers' gains optimal setting unit receives from the MPC policy selection unit the plan with the selected MPC policies, and based on each policy (or strategy) it calls the model (or the transfer function) of that configuration and manipulates it in the optimization process. The output is the controllers' gains for each strategy based on the given manufacturing system's parameters and aligned with the market strategy.

\section{MPC demand satisfaction check unit}

This layer is actually responsible for checking that the current production-rate or inventory level satisfies the required demandand this is why it takes place on-line. The check is based on comparing the current production level with the required capacity rate, the current WIP level with the ideal WIP level, and the current inventory level with the target inventory level based on which MPC policy is being adopted. These reference levels are calculated based on the anticipated demand, and thus, meeting these levels means satisfying the market demand.

Based on the discrepancy between the compared levels, a decision is made to compensate for that discrepancy through the previously calculated control gain values. The decision indicates which gain is to be implemented and for how long in order to meet the required level. This process is carried out on an interactive basis with the operational level. The manufacturing system updates this unit in the DLU with the current status of the system, and based on the previously fed data of the demand, a control action is decided. Thus, this unit is mainly responsible for what is known in the literature of MPC as production control. Sudden changes in demand are accounted for through this unit through the continuous update of the required inventory, WIP, and capacity levels based on the current demand. The flowchart for the algorithms of the different units in the DLU architecture is shown in Appendix A.

\section{Application and analysis of the developed agile control approach}

\subsection{Application to automatic PCB assembly factory}

Next, application of the agile MPC system that has been developed will be illustrated using a real industrial case study 
a

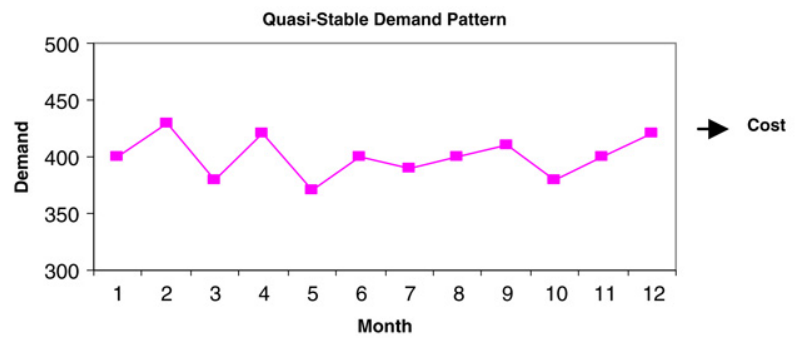

b

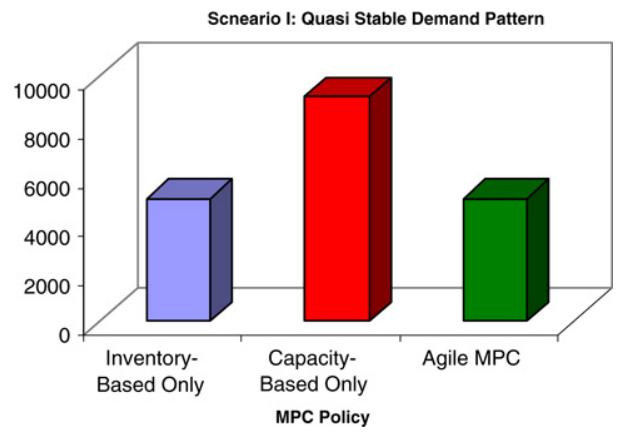

Fig. 3. (a) Quasi-stable demand pattern (b) Cost of different MPC policies with quasi-stable demand pattern.

a

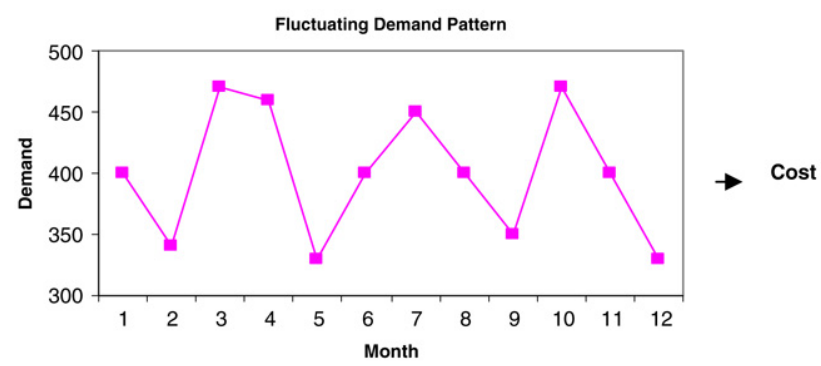

b

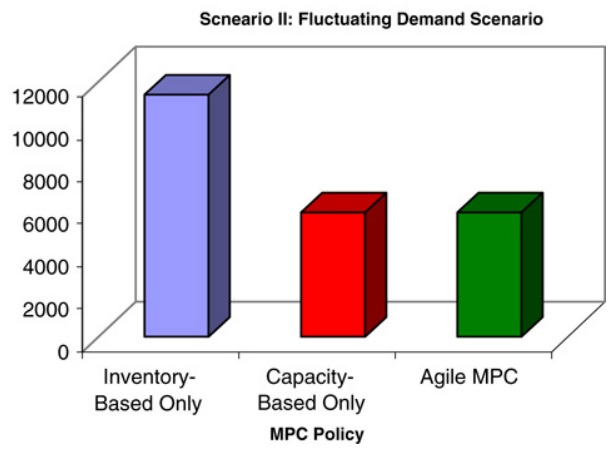

Fig. 4. (a) Fluctuating demand pattern (b) Cost of different MPC policies with fluctuating demand pattern.

in an automatic PCB assembly factory. The objective of this case study is to highlight the use of the developed approach in a very turbulent market that can resemble the agile environment, which is the electronics market, and in a manufacturing system that is an ideal candidate for agile manufacturing, which is the automatic PCB assembly line. The product in this case is the random access memory (RAM) module. The full data for the case study are provided in Appendix B.

Table 2 displays a summary of all of the deliverables of the designed DLU or the supervisory control. The first two rows show the monthly demand data, while the third row shows the different MPC policies applied during these months with the optimal controllers' gains for each policy (calculated off-line). The rest of the table shows the on-line production control carried out by the third layer to meet these monthly demands.

\subsection{Comparative cost analysis}

A cost analysis comparison between different policies is conducted to show how each policy, including the agile MPC developed, can handle different demand scenarios for the same discussed industrial case. The objective of this comparison is to highlight the efficiency of the developed agile MPC approach and its superiority in dealing with mixed-demand patterns.

The policies considered are inventory-based MPC policy, capacity-based MPC policy, and finally, the agile MPC policy (that can adopt both policies when needed). The demand patterns investigated are quasi-stable demand (demand with small fluctuations), fluctuating demand, and demand patterns that are a combination of these two types of demands.

The cost calculations in this paper are roughly estimated based on real data from the same case study to give indication about the efficiency of each policy. However, full management accounting is beyond the scope of this paper. The cost data for the each policy are provided in Appendix C. Based on the given data for both policies, the following cost parameters are calculated to be used later in the analysis for each policy with different demands:

For the capacity-based MPC policy, the costs encountered are:

- Monthly cost for capacity scalability (cost of added machines).

- Monthly cost of underutilized capacity.

For the inventory-based MPC policy, the costs encountered are:

- The holding cost $\left(C_{H}\right)$, which will be calculated by first calculating the quantity of unsold RAM/month $\left(Q_{H}\right)$ and then multiplying this quantity by the holding cost using the following equations:

$$
\begin{aligned}
& Q_{H}=\text { Production }- \text { Demand } \\
& C_{H}=Q_{H} * i * \operatorname{Pr} .
\end{aligned}
$$

- The backlog cost $\left(C_{B}\right)$ will be calculated for each month first by calculating the backlog quantity $\left(Q_{B}\right)$ and then multiplying this quantity by the backlog penalty $\left(P_{B}\right)$ and the cost of loss of good will $\left(C_{\mathrm{LGW}}\right)$, as shown in the following equations:

$$
\begin{aligned}
& Q_{B}=\text { Demand }-\left(\text { Production }+Q_{H}\right) \\
& C_{B}=Q_{B} *\left(P_{B}+C_{\mathrm{LGW}}\right) * P_{S} .
\end{aligned}
$$

For the agile MPC policy, the costs encountered are:

The developed agile MPC approach has the ability to deal with all demand patterns through combining the previous two policies and applying each one to its suitable demand pattern. Thus, in this analysis, in quasi-stable demand patterns the agile MPC policy will adopt the inventory-based MPC policy. In fluctuating demand, the capacity-based MPC policy will be adopted. Finally, in mixed demand, a mix between the two policies will be used in accordance with the demand segmentation approach discussed earlier.

The demand patterns considered and their values are shown in Figs. 3(a), 4(a), and 5(a). It is important to note that the mixeddemand patterns in Fig. 5 will be divided by the first layer in the DLU in the agile MPC policy into three zones. The first zone is from months 1 to 3 and will adopt the inventory-based policy because 


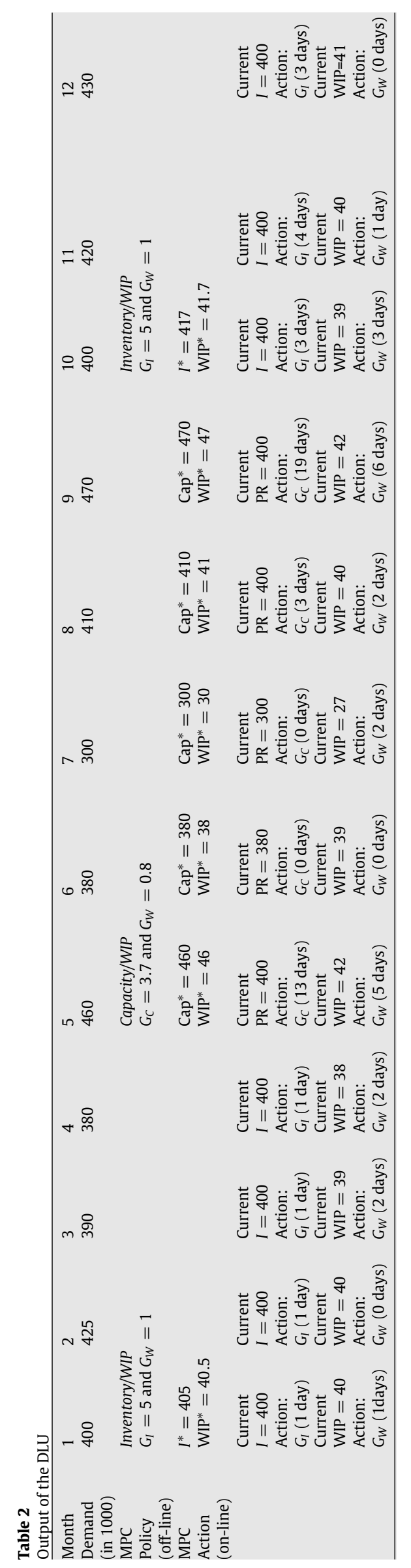


a

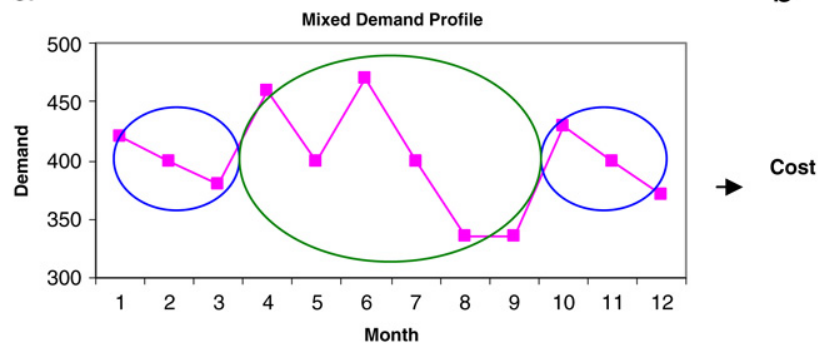

b

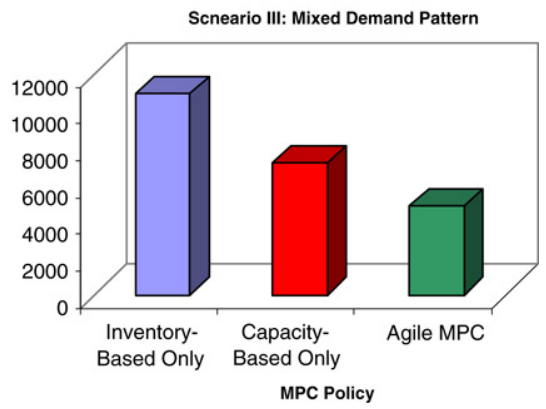

Fig. 5. (a) Mixed-demand pattern (b) Cost of different MPC policies with mixed-demand pattern.

it has a quasi-stable trend. The second zone will be from months 4 to 9 and will adopt a capacity-based policy due to demand fluctuations. The last zone will be from months 10 to 12 and will again adopt an inventory-based policy for the same reasons as for the first zone. Figs. 3(b), 4(b), and 5(b) plot the overall costs over the 12 months for each MPC policy with the three considered demand patterns.

Analysis of these figures leads to the following observations:

- With quasi-stable demand, inventory-based MPC policy shows a better performance in terms of cost because the variations of demand from the target inventory level are limited, and thus the holding cost as well as the backlog cost is minimal. As for the capacity-based policy, the cost to handle that demand pattern is quite a bit higher because the capacity is usually scaled to high values that need high-demand variation to avoid paying for underutilized capacity or capacity loss, as in this case.

- With fluctuating demand, the opposite scenario was found, where the capacity-based MPC policy showed a better cost performance in handling this kind of demand. The reason for that is the huge variation in demand values, which justifies the use of extra capacity (capacity scalability) in cases of demand increase. At the same time, these demand variations lead to high levels of accumulated inventory (holding cost) and sometimes a shortage in the level of available inventory (backlog cost), leading to higher cost for the inventory-based policy.

- With fluctuating demand, the opposite scenario was found, where the capacity-based MPC policy showed a better cost performance in handling this kind of demand. The reason for that is the huge variation in demand values, which justifies the use of extra capacity (capacity scalability) in cases of demand increase. At the same time, these demand variations lead to high levels of accumulated inventory (holding cost) and sometimes a shortage in the level of available inventory (backlog cost), leading to higher cost for the inventory-based policy.

- The developed agile MPC approach showed the best performance of the three considered demand patterns. In a quasistable demand pattern, the agile MPC approach adopted an inventory-based policy by engaging the inventory controller, and this is why it was as cost efficient as the classical inventorybased policy. In fluctuating demand, the DLU disengaged the inventory control and switched to the capacity controller to have the same cost-effective performance as the typical capacitybased MPC policy. However, in the mixed-demand pattern, the agile MPC approach was far superior to the other two policies due to its ability to handle each period in the demand pattern with the suitable policy, manipulating its switching ability between different distributed controllers.

\section{Conclusions}

A new approach was proposed for how enterprises can maintain their agility through enabling manufacturing planning and control (MPC) systems to adopt different policies and align with the current market strategy. A dynamic model for the agile MPC system was presented, where different MPC policies can be adopted through distributed controllers for each policy and a supervisory controller responsible to handle these controllers through a decision logic unit (DLU). A multi-layer architecture for this DLU was developed. The first layer in the DLU is responsible for managing the switching protocol among the distributed controllers based on the market strategy. The optimal settings of these controllers' gains require a trade-off between the responsiveness level and the cost of deviating from the target production based on the market competitiveness plan adopted by the higher management level. This decision is carried out through a multi-objective optimization technique in the second layer of the DLU. The last layer works on-line with the operational level to control production, WIP, and inventory levels in accordance with the marketing strategy using MPC policy and settings indicated by the previous two layers.

To demonstrate the applicability of the developed approach, it was applied to an industrial case study for automatic PCB assembly. The output of the DLU unit showed how the developed agile MPC system was able to control the assembly line to align with the marketing strategy and meet the required demand. Furthermore, the comparative cost analysis conducted verified the fundamental philosophy of the agile MPC system proposed in this paper by showing that, in a typical dynamic market environment, the MPC system can maintain its agility by the ability to efficiently react to different anticipated demand patterns through different distributed controllers for the main manufacturing parameters.

The presented work offers a starting point for more investigation about the need to close the gap between the marketing level and the operational level in agile enterprises. Further work is required to explore other dynamical approaches to achieve that integration in this dynamic environment.

\section{Appendix A. DLU algorithm}

The flowchart for the architecture algorithm of the developed DLU is shown in Fig. A.1. Each of the three layers of the DLU is represented by one of the three columns, respectively. The variables in Fig. A.1 are given as follows:

$n=$ number of months in the anticipated demand

MS = market sensitivity

$\alpha=$ weights assigned by management

$\omega_{n}=$ natural frequency

$\zeta=$ damping ratio

$t_{r}=$ rise time

$\% O S=$ percentage overshoot. 


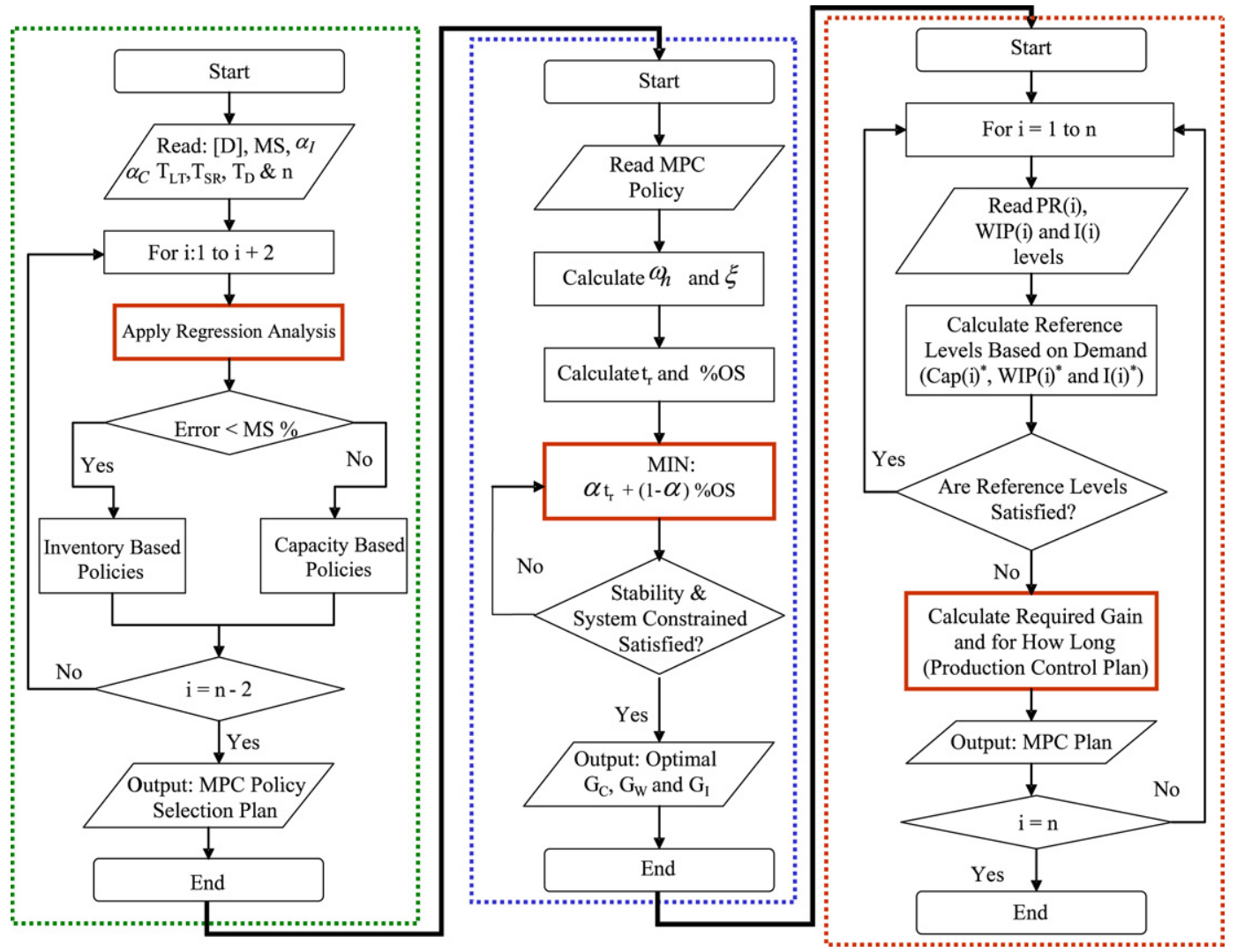

Fig. A.1. Flowchart for the architecture algorithm of the developed DLU.

\section{Appendix B. Data for the case study}

\section{Market data}

See Table B.1.

2-Marketing strategy for each MPC policy (weights for the $\mathrm{MOO})$ :

\begin{tabular}{lll} 
MPC policy & $\begin{array}{l}\text { Responsiveness } \\
\text { objective }\end{array}$ & $\begin{array}{l}\text { Cost } \\
\text { objective }\end{array}$ \\
\hline Inventory/WIP $\left(\alpha_{I}\right)$ & 0.3 & 0.7 \\
Capacity/WIP $\left(\alpha_{C}\right)$ & 0.7 & 0.3
\end{tabular}

3-Market Sensitivity (regression error): $10 \%$.

\section{System data}

\section{1-Time Parameters:}

- Production lead time $T_{\mathrm{LT}}$ : 2 days

- Capacity scalability delay time $T_{D}$ : 3 days

- Shipment time $T_{\mathrm{SR}}$ : 5 days.

2-System Throughput: 400K RAM/month (20K RAM/day) 3-System Limits:

- Capacity: The shop floor of the factory is composed of two lines; each line contains four pick-and-place machines. The pick-andplace machines are of two types (two of each per line): one type is a chip shooter type (high capacity) with average production rate of $3.2 \mathrm{~K} /$ day and another type (medium capacity) with average production rate of $1.8 \mathrm{~K} /$ day. Due to space limitations of the shop floor, only one pick-and-place machine of the medium capacity type can be added for each of the assembly lines. Thus, the maximum capacity rate that can be added to the factory is 3.6K/day.

- Maximum input rate increase: 6K RAM/day

- Maximum WIP rate increase: 1K RAM/day.

\section{Appendix C. Data for comparative cost study}

\section{Capacity-based MPC case cost calculations}

The monthly cost for capacity scalability is calculated using Capital Recovery Analysis [9]. The input data for this analysis are as follows:

- The capital cost $(P)$ for the smaller $\mathrm{m} / \mathrm{c}$ (1.8K capacity) is $\$ 100,000$.

- The interest rate (i) is $1 \%$ accumulated monthly.

- Depreciation period $(N)$ is 8 years.

- Salvage value $(D)$ will be equal to $10 \%$ of the capital cost and the declining balance method will be used to calculate the salvage value.

The monthly cost $(A)$ for having the smaller pick-and-place (1.8 K capacity) machine will be calculated through adding the capital recovery cost minus the sinking found factor as shown in Eq. (C.1):

$\left.\left.A=\left[P \frac{i(1+i)^{N}}{(1+i)^{N}-1}\right)\right]-\left[P(1-D)^{N} \frac{i}{(1+i)^{N}-1}\right)\right]$.

From the previous data and using Eq. (C.1), the monthly cost of this machine will be $A=\$ 1300$.

The other cost parameter that should be considered in the capacity scalability cost analysis is the underutilized capacity cost, or sometimes referred to as capacity loss cost. Although there is no well-accredited or standard formula for that cost, an accepted assumption would be treating the underutilized capacity cost as a holding cost where the unused capacity portion is paid for as a function of the overall cost of the capacity unit. For example, in this case, if the monthly capacity scalability cost of adding a pick-and-place machine is $\$ 1300$, and the utilized capacity of this machine is only $75 \%$ of the overall capacity, then the monthly cost of underutilized capacity would be: $(1300 / 4)=\$ 325$. 
Table B.1

1-RAM Monthly Demand

\begin{tabular}{|c|c|c|c|c|c|c|c|c|c|c|c|c|}
\hline Month & 1 & 2 & 3 & 4 & 5 & 6 & 7 & 8 & 9 & 10 & 11 & 12 \\
\hline Demand (in 1000) & 400 & 425 & 390 & 410 & 460 & 380 & 300 & 410 & 470 & 400 & 420 & 430 \\
\hline
\end{tabular}

\section{Inventory-based MPC case cost calculations}

In any inventory cost analysis, there are two important cost parameters that should be considered. First is the holding cost and second is the stock-out cost or backlog cost. Each of these cost parameters will be calculated using the following data:

- Monthly interest rate for held inventory items (i) is $0.2 \%$ (typical value in low-interest inventory cases) [18]. It is important to note here that this interest value plays a very important role in such cost analyses. Thus, the analysis can be highly altered if this value changes. However, the effect of interest-rate variation is a wide research area in the field of economics and beyond the scope of this paper.

- Price of the RAM (Pr) is equal to the manufacturing cost plus the components. The manufacturing cost can be calculated by dividing the monthly production with monthly cost: Mfg. Cost (the latter is calculated using Eq. (C.1)) $=400,000 / 15,200=$ $\$ 26 /$ RAM. The component cost based on the priced bill of materials (BOM) is approximately $\$ 4 /$ RAM. Thus, the cost of the RAM, $P r=\$ 30$.

- The average selling price $\left(P_{S}\right)$ of the considered RAM module is $\$ 100$.

- Based on the market strategy and customer contracts, the penalty for instantaneous unmet demand or backlog $\left(P_{B}\right)$ for each RAM module is $0.01 \%$ of the selling price.

- Based on the market competitiveness estimations, the estimated cost for loss of good will $\left(C_{\mathrm{LGW}}\right)$ for unmet demand is also $0.01 \%$ of the selling price.

- The reference inventory level will be calculated using the classical approach of summing all of the anticipated demands over the year and then dividing the total by 12 to have the monthly inventory level: $I^{*}=\frac{\sum \text { Demand }}{12}$.

\section{References}

[1] Anderson JC, Cleveland G, Schroeder RG. Operations strategy: A literature review. Journal of Operations Management 1989;8(2):133-58.

[2] Bruccoleri M, Amico M, Perrone G. Distributed intelligent control of exceptions in reconfigurable manufacturing systems. International Journal of Production Research 2003;41(7):1393-412.

[3] Bruccoleri M, Renna P, Perrone G. Reconfiguration: A key to handle exceptions and performance deteriorations in manufacturing operations. Int'l Journal of Production Research 2005;43(19):4125-45.

[4] Deif A, ElMaraghy W. Effect of time-based parameters on the agility of a dynamic MPC system. Annals of the CIRP 2006;55(1):437-40.

[5] Dejonckheere J, Diseny S, Lambrecht M, Towill D. Dynamics of aggregate planning. Journal of Production and Planning Control 2003;14(6):497-516.

[6] Diseny S, Towill D. A discrete transfer function model to determine the dynamic stability of a vendor managed inventory in supply chain. Int'l Journal of Production Research 2002;40(1):179-204.

[7] Duffie N, Prabhu V, Kaltjob P. Closed-loop real-time cooperative decisionmaking dynamics in heterarchical manufacturing systems. Journal of Manufacturing Systems 2002;21(6):409-18.

[8] Duffie N, Falu I. Control-theoretic analysis of a closed loop PPC system. Annals of the CIRP 2002;52(1):379-82.

[9] Fraser NM, Bernhardt I, Jewkes EM, Tajima M. Engineering economics in Canada. Toronto: Pearson Education Canada Inc.; 2006.

[10] Hayes RH, Schmenner RW. How should you organize manufacturing? Harvard Business Review 1978;56(1):105-18.
[11] Hayes RH, Wheelwright SC. Restoring our competitive edge. New York: John Wiley and Sons; 1984.

[12] Ioannidis S, Tsourveloudis NC, Valavanis K. Fuzzy supervisory control of manufacturing systems. IEEE Transactions on Robotics and Automation 2004; 20(3):379-89.

[13] John S, Towill DR, Naim M. Dynamic analysis of a WIP compensated support system. Int'l Journal of Manufacturing System Design 1994;1:283-97.

[14] Kim J-H, Duffie N. Backlog control design for a closed loop PPC system. Annals of the CIRP 2004;54(1):456-9.

[15] Kim J-H, Duffie N. Design and analysis for a closed loop capacity control of a multi workstation production system. Annals of the CIRP 2005;55(1): $470-474$.

[16] Kotha S, Swamidass PM. Strategy, advanced manufacturing technology, and performance: Empirical evidence from US manufacturing firms. Journal of Operations Management 2000;8(3):257-77.

[17] Moreno-Lizaranzu M, Wysk R, Hong J, Prabhu V. A hybrid shop-floor control system for food manufacturing. IIE Transactions 2001;33(3):193-202.

[18] Nahmias S. Production and operation analysis. 5 ed. McGraw-Hill; 2001.

[19] Nise N. Control systems engineering. New York: John Wiley and Sons; 2000.

[20] Nyhuis P, Logistic operating curves-a comprehensive method for rating logistic potentials. EURO XIII/OR36; 1994.

[21] Prabhu VV. Stable fault adaptation in distributed control of heterarchical manufacturing job shops. IEEE Transactions on Robotics and Automation 2003;19(1):142-9.

[22] Ratering A, Duffie N. Design and analysis of closed-loop single-workstation PPC system. Annals of the CIRP 2003;52(1):375-9.

[23] Skinner W. Manufacturing: The formidable competitive weapon. New York: John Wiley and Sons; 1985

[24] Towill D, Evans G, Cheema P. Analysis of an adaptive reasonable inventory control system. Journal of Production Planning and Control 1997;8(6): 545-557.

[25] Tsourveloudis N, Valavanis K. On the measurement of enterprise agility. Journal of Intelligent and Robotic Systems 2002;33:329-42.

[26] Wacker JG, Hanson M. Some practical advice to manufacturing managers: Empirical results from the Global Manufacturing Research Group. Production and Inventory Management Journal 1997;38(3):64-72.

[27] Wiendahl HP. Load oriented manufacturing control. Hanover, NH: SpringerVerlag; 1995.

[28] Wiendahl HP, Breithaupt J. Modeling and controlling of dynamics of production system. Journal of Production Planning and Control 1999;10(4): 389-401.

[29] Wiendahl HP, Breithaupt J. Automatic production control applying control theory. Int'l Journal of Production Economics 2000;63(1):33-46.

[30] Wikner J., Dynamic modeling and analysis of information flows in productioninventory and supply chain systems. Pro. 1 Series No 12. Sweden: Linkoping Institute of Technology; 1994.

[31] Wysk RA, Smith JS. A formal functional characterization of shop floor control. Computers and Industrial Engineering 1995;28(3):631-43.

Dr. Ahmed M. Deif received his Ph.D. and MASc in industrial and manufacturing systems engineering from the University of Windsor in 2006 and 2003, respectively. He received his B.Sc. from The American University in Cairo (1999). He currently is Assistant Professor, Dept. of Industrial Manufacturing Systems, University of Regina, Regina, Canada. His research interests are in manufacturing capacity management, manufacturing planning and control, and dynamic analysis of manufacturing systems. He is a research affiliate of the International Academy for Production Engineering Research (CIRP).

Dr. Waguih H. ElMaraghy is a professor of engineering and director of the Intelligent Manufacturing Systems (IMS) Centre, Dept. of Industrial and Manufacturing Systems Engineering, University of Windsor. He is also a senior member of the Society of Manufacturing Engineers, the Society of Automotive Engineers, a fellow of the Canadian Society for Mechanical Engineering, a fellow of the American Society of Mechanical Engineers, and a fellow member of the International Academy for Production Engineering Research (CIRP). Dr. ElMaraghy is an active researcher and author with expertise in engineering design, manufacturing automation, and industrial productivity. 